\title{
A Note on Two Multicolor Ramsey Numbers
}

\author{
Alexander Engström * \\ Institute of Theoretical Computer Science \\ ETH Zürich, Zürich, Switzerland \\ engstroa@inf.ethz.ch
}

Submitted: May 3, 2005; Accepted: Aug 19, 2005; Published: Aug 30, 2005

Mathematics Subject Classifications: 05C55

\begin{abstract}
Two new bounds for multicolor Ramsey numbers are proved:

$R\left(K_{3}, K_{3}, C_{4}, C_{4}\right) \geq 27$ and $R_{4}\left(C_{4}\right) \leq 19$.
\end{abstract}

\section{Introduction}

We prove two new bounds for multicolor Ramsey numbers, a lower bound for $R\left(K_{3}, K_{3}, C_{4}, C_{4}\right)$ by coloring $K_{26}$, and an upper bound for $R_{4}\left(C_{4}\right)$ by a density argument.

\section{The Ramsey number $R\left(K_{3}, K_{3}, C_{4}, C_{4}\right)$}

From the survey of Ramsey numbers by Radziszowski [3] we know that $R\left(K_{3}, K_{3}, C_{4}, C_{4}\right) \geq 26$. We use $C_{5}$-decompositions to construct a four-coloring of the edges of $K_{26}$, which show that $R\left(K_{3}, K_{3}, C_{4}, C_{4}\right) \geq 27$. The technique used in this section was invented by Exoo and Reynolds [2].

Theorem $1 R\left(K_{3}, K_{3}, C_{4}, C_{4}\right) \geq 27$.

Proof: Let $X, Y, I, \overline{0}$, and $\overline{1}$ be defined by

$$
X=\left[\begin{array}{lllll}
0 & 1 & 0 & 0 & 1 \\
1 & 0 & 1 & 0 & 0 \\
0 & 1 & 0 & 1 & 0 \\
0 & 0 & 1 & 0 & 1 \\
1 & 0 & 0 & 1 & 0
\end{array}\right] Y=\left[\begin{array}{lllll}
0 & 0 & 1 & 1 & 0 \\
0 & 0 & 0 & 1 & 1 \\
1 & 0 & 0 & 0 & 1 \\
1 & 1 & 0 & 0 & 0 \\
0 & 1 & 1 & 0 & 0
\end{array}\right] I=\left[\begin{array}{lllll}
1 & 0 & 0 & 0 & 0 \\
0 & 1 & 0 & 0 & 0 \\
0 & 0 & 1 & 0 & 0 \\
0 & 0 & 0 & 1 & 0 \\
0 & 0 & 0 & 0 & 1
\end{array}\right] \overline{0}=\left[\begin{array}{l}
0 \\
0 \\
0 \\
0 \\
0
\end{array}\right] \overline{1}=\left[\begin{array}{l}
1 \\
1 \\
1 \\
1 \\
1
\end{array}\right]
$$

*Research supported by ETH and Swiss National Science Foundation Grant PP002-102738/1 
The critical colorings which show that $R\left(K_{3}, K_{3}\right)>5$ and $R\left(C_{4}, C_{4}\right)>5$ have the adjacency matrices $X$ and $Y$. Observe that $X+Y+I$ is the all-ones $5 \times 5$ matrix.

We now construct four $26 \times 26$ adjacency matrices $M_{i}$, so that $M_{1}$ and $M_{2}$ contain no $K_{3}$, and $M_{3}$ and $M_{4}$ contain no $C_{4}$.

Given a triangle-free graph on $n$ vertices, one can construct a triangle-free graph on $n m$ vertices by replacing each vertex with $m$ vertices and each edge with $K_{m, m}$. We construct the two first graphs, which are isomorphic, by beginning with $C_{5}$, replacing the edges with $K_{5,5}-e$, and then adding a vertex with five edges.

$$
M_{1}=\left[\begin{array}{cccccc}
0 & X & X & X & X & \overline{1} \\
X & X & X & X & X & \overline{0} \\
X & X & X & X & X & \overline{0} \\
X & X & X & X & X & \overline{0} \\
X & X & X & X & X & \overline{0} \\
\overline{1}^{T} & \overline{0}^{T} & \overline{0}^{T} & \overline{0}^{T} & \overline{0}^{T} & 0
\end{array}\right] \quad M_{2}=\left[\begin{array}{cccccc}
Y & Y & Y & Y & Y & \overline{0} \\
Y & Y & Y & Y & Y & \overline{0} \\
Y & Y & Y & Y & Y & \overline{0} \\
Y & Y & Y & Y & Y & \overline{0} \\
Y & Y & Y & Y & 0 & \overline{1} \\
\overline{0}^{T} & \overline{0}^{T} & \overline{0}^{T} & \overline{0}^{T} & \overline{1}^{T} & 0
\end{array}\right]
$$
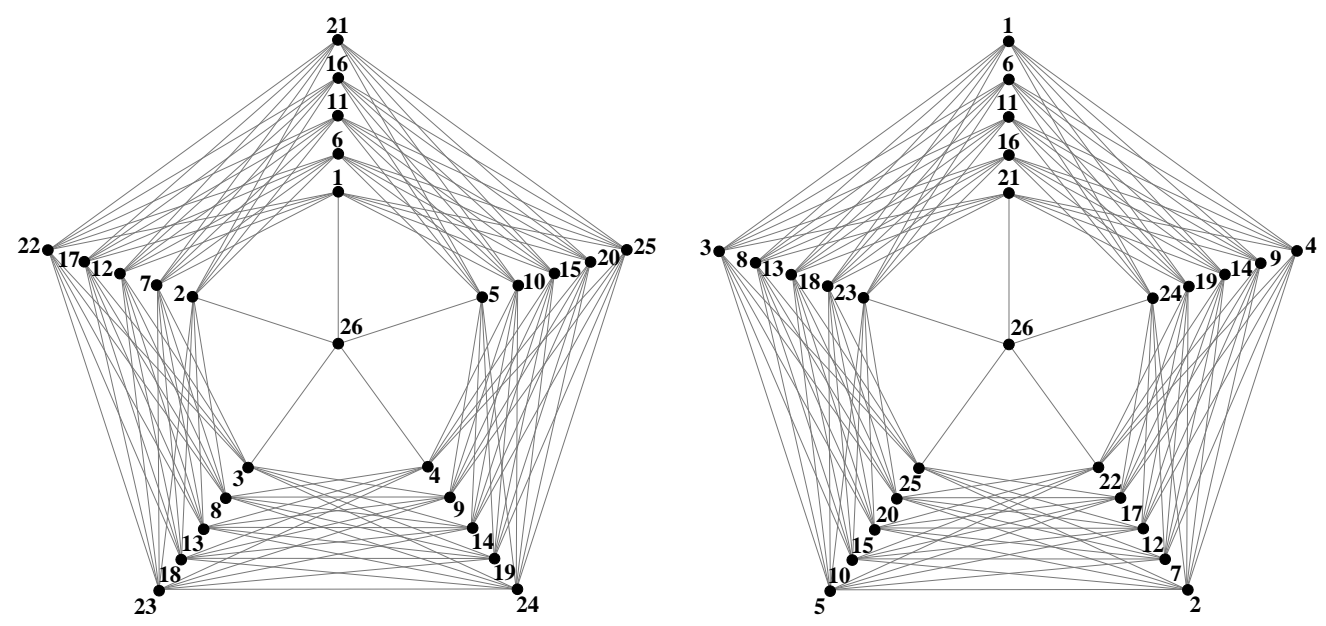

Figure 1: The graphs with adjacency matrices $M_{1}$ and $M_{2}$.

We denote the vertices from the top of the matrices as $1,2, \ldots 26$. The graphs are shown in Figure 1. Vertices $1-25$ are the triangle-free constructions from $C_{5}$ and $K_{5,5}-e$. Vertex 26 is in no triangle, since its neighbors have no edges between them. Hence, the graphs are triangle-free.

The remaining edges are distributed as described by the adjacency matrices $M_{3}$ and $M_{4}$.

$$
M_{3}=\left[\begin{array}{cccccc}
X & I & 0 & 0 & I & \overline{0} \\
I & 0 & I & 0 & 0 & \overline{0} \\
0 & I & 0 & I & 0 & \overline{1} \\
0 & 0 & I & 0 & I & \overline{1} \\
I & 0 & 0 & I & 0 & \overline{0} \\
\overline{0}^{T} & \overline{0}^{T} & \overline{1}^{T} & \overline{1}^{T} & \overline{0}^{T} & 0
\end{array}\right] \quad M_{4}=\left[\begin{array}{cccccc}
0 & 0 & I & I & 0 & \overline{0} \\
0 & 0 & 0 & I & I & \overline{1} \\
I & 0 & 0 & 0 & I & \overline{0} \\
I & I & 0 & 0 & 0 & \overline{0} \\
0 & I & I & 0 & Y & \overline{0} \\
\overline{0}^{T} & \overline{1}^{T} & \overline{0}^{T} & \overline{0}^{T} & \overline{0}^{T} & 0
\end{array}\right]
$$



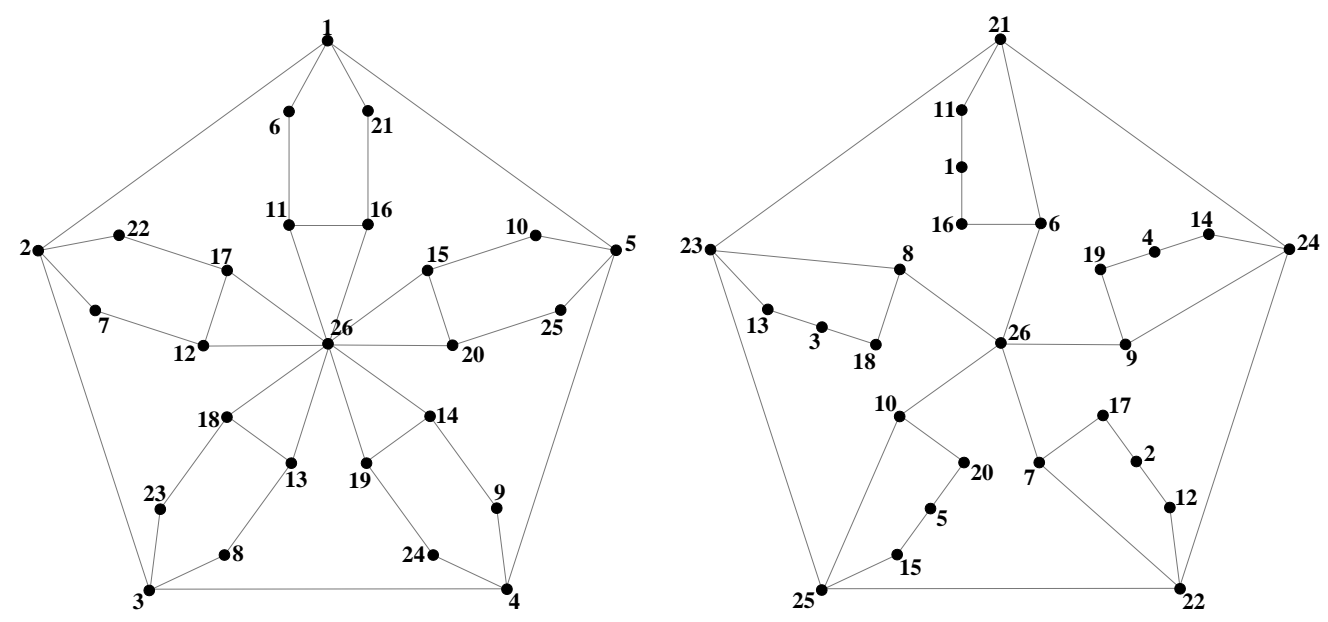

Figure 2: The graphs with adjacency matrices $M_{3}$ and $M_{4}$.

It is not hard to see that $M_{1}+M_{2}+M_{3}+M_{4}$ is the adjacency matrix of $K_{26}$. It is clear from Figure 2 that there are no quadrilaterals.

\section{The Ramsey number $R_{4}\left(C_{4}\right)$}

From the Ramsey number survey [3] we also know that $18 \leq R_{4}\left(C_{4}\right) \leq 21$. It was shown by Clapham, Flockhart and Sheehan [1] that a $C_{4}$-free graph with 19 vertices has at most 42 edges. Since $4 \cdot 42=168$ and there are 171 edges in $K_{19}$, it is not possible to four-color the edges of $K_{19}$ without a monochromatic quadrilateral.

Theorem $2 \quad R_{4}\left(C_{4}\right) \leq 19$.

\section{References}

[1] C.R.J. Clapham, A. Flockhart, J. Sheehan, Graphs without four-cycles, J. Graph Theory, 13 (1989) 29-47.

[2] G. Exoo, D.F. Reynolds, Ramsey numbers based on $C_{5}$-decompositions, Discrete Math., 71 (1988) 119-127.

[3] S. Radziszowski, Small Ramsey numbers, Electron. J. Combin., DS1 (revision \#9, 2002). 